\title{
Central Femoral Head Chondromalacia Is Associated with a Diagnosis of Hip Instability
}

\author{
W. Michael Pullen, M.D., Daniel M. Curtis, M.D., and Marc R. Safran, M.D.
}

Purpose: To compare the locations and patterns of femoral head chondral damage in patients with instability in contrast to those with femoroacetabular impingement (FAI) without instability. Methods: All consecutive hip arthroscopies were reviewed from 2013 to 2020 from a single surgeon. Intraoperative records were reviewed on all patients identified to have femoral head chondromalacia. Data were collected to include laterality, location of femoral head chondromalacia, intraoperative diagnosis (instability and/or FAI subtype), and ease of distractibility. The location of the femoral head chondromalacia was defined on the basis of intraoperative description. Chi-squared and Fisher's exact tests were used for categorical variables, and a two-sample $t$ test was used for continuous variables. Statistical significance was set at $P<.05$. Results: A total of 64 patients were in the study cohort, with 32 patients identified as having non-central head chondromalacia and 32 patients identified as having central head chondromalacia. Of the patients with central head chondromalacia, $81 \%$ were diagnosed with instability. Central head chondromalacia was associated with a sensitivity of $84 \%(71 \%-97 \%)$, specificity of $82 \%(69 \%-95 \%)$, and positive predictive value of $81 \%(67 \%-95 \%)$. Conclusions: A high percentage of patients with central femoral head chondromalacia were found to have hip microinstability. These results suggest that there is a pattern of femoral head chondral damage in patients with hip microinstability. Level of Evidence: Level III, case-control study.

\section{Introduction}

$\mathbf{H}$ ip microinstability is an increasingly accepted cause of young, nonarthritic hip pain. ${ }^{1-4}$ Initially an ill-defined and underappreciated clinical entity, an emerging body of evidence has sought to better define hip microinstability. ${ }^{2,4,5}$ Patients with hip microinstability may experience hip joint pain, unsteadiness, or apprehension. Moreover, good results have been

From the Medical University of South Carolina, Department of Orthopaedics and Physical Medicine, Charleston, South Carolina, U.S.A. (W.M.P.); Reno Orthopaedic Clinic, Reno, Nevada, U.S.A. (D.M.C.); and Stanford University, Department of Orthopaedic Surgery, Redwood City, California, U.S.A. (M.R.S.).

The authors report the following potential conflicts of interest or sources of funding: M.R.S. reports personal fees from Smith $\theta$ Nephew, Medacta, DJO, Stryker, Elsevier, and Lippincott. He is an unpaid consultant and has received stock options from Biomimedica, all outside the submitted work. Full ICMJE author disclosure forms are available for this article online, as supplementary material.

Received July 12, 2021; accepted October 22, 2021.

Address correspondence to W. Michael Pullen, M.D., Medical University of South Carolina Department of Orthopaedics and Physical Medicine, 96 Jonathan Lucas St., Suite 708, MSC 622, Charleston, SC 29425, U.S.A. E-mail: pullenwi@musc.edu

(C) 2021 THE AUTHORS. Published by Elsevier Inc. on behalf of the Arthroscopy Association of North America. This is an open access article under the CC BY-NC-ND license (http://creativecommons.org/licenses/by-nc-nd/4.0/). 2666-061X/21949

https://doi.org/10.1016/j.asmr.2021.10.023 achieved through identifying and treating patients with instability using capsular plication techniques. ${ }^{6,7}$

Microinstability must be distinguished from general soft tissue laxity similar to other joints (i.e., shoulder, patellofemoral) where supraphysiological motion must also be associated with pain or dysfunction. Although this can develop in a post-traumatic or postsurgical state, it can also be present in a native hip secondary to bony deficiency and/or soft tissue insufficiency. ${ }^{2,8}$ Making the diagnosis of microinstability is based on a multitude of factors that are identified through a thorough history and physical exam, imaging studies, and intraoperative findings. ${ }^{1,2,4,5,9,10}$ Moreover, the diagnosis of hip microinstability can occur with normal radiographs or be a concurrent diagnosis with labral injuries, femoroacetabular impingement (FAI), hip dysplasia or borderline dysplasia. Therefore, a high index of suspicion is necessary to make the diagnosis. Toward that end, identification of intraoperative features associated with hip instability could be beneficial to the surgeon to maximize surgical outcomes.

Supraphysiological motion of the hip may result in characteristic wear or damage patterns on the femoral head, acetabulum, or labrum. ${ }^{5}$ Cadaveric models have demonstrated that the central femoral head moves relative to the acetabulum in all planes of motion at extremes of motion. ${ }^{11-13}$ Moreover, femoral head 


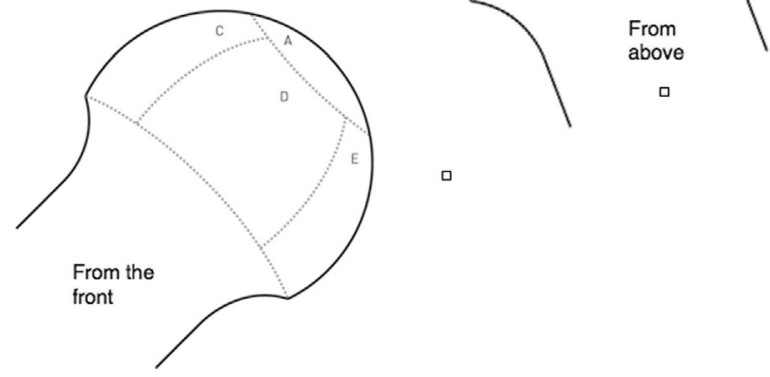

Fig 1. Illustration of zones of femoral head chondromalacia when viewed arthroscopically, demonstrating central (A), posterior (B), lateral (C), anterior (D), and medial (E) chondromalacia.

motion and subluxation have been identified in asymptomatic ballet dancers, further confirming supraphysiological motion of the hip. ${ }^{14-17}$ The increased motion of the central femoral head may place this region at particular risk in patients with instability, as the femoral head may translate to, or over, the edge of the acetabular rim, potentially resulting in a shear injury to the central femoral head articular cartilage. The purpose of this study was to compare the locations and patterns of femoral head chondral damage in patients with hip microinstability, in contrast to those with FAI without instability. It is our hypothesis that patients with microinstability will demonstrate increased central femoral head chondromalacia (CM) compared with patients without microinstability.

\section{Methods}

After institutional review board approval, a retrospective review identified hip arthroscopies performed by the senior author from 2013 to 2020 . A search was conducted for the diagnosis of femoral head chondromalacia within the operative record, identifying hip arthroscopies, which included this finding. Inclusion criteria included hip arthroscopy for FAI and/or instability, which specified the location of femoral head chondromalacia. Exclusion criteria included hip arthroscopy performed for non-FAI and/or instability pathology or incomplete records. The diagnosis of microinstability was established on the basis of previously defined characteristics to include intra-articular hip pain and laxity of the hip joint intraoperatively. ${ }^{5}$ Medical records and operative records were then reviewed to obtain patient demographic information to include gender, BMI, age, and race. Operative reports were reviewed and data were collected to include laterality, location of femoral head chondromalacia, intraoperative diagnosis (instability and/or FAI subtype), and ease of distractibility (number of turns to distract, residual subluxation, distraction with body weight). The location of the femoral head chondromalacia was defined on the basis of intraoperative description, which was based on an intraoperative recording sheet (Fig 1). Intraoperative viewing was performed through both anterolateral and modified anterior portals, as well as a posterolateral portal
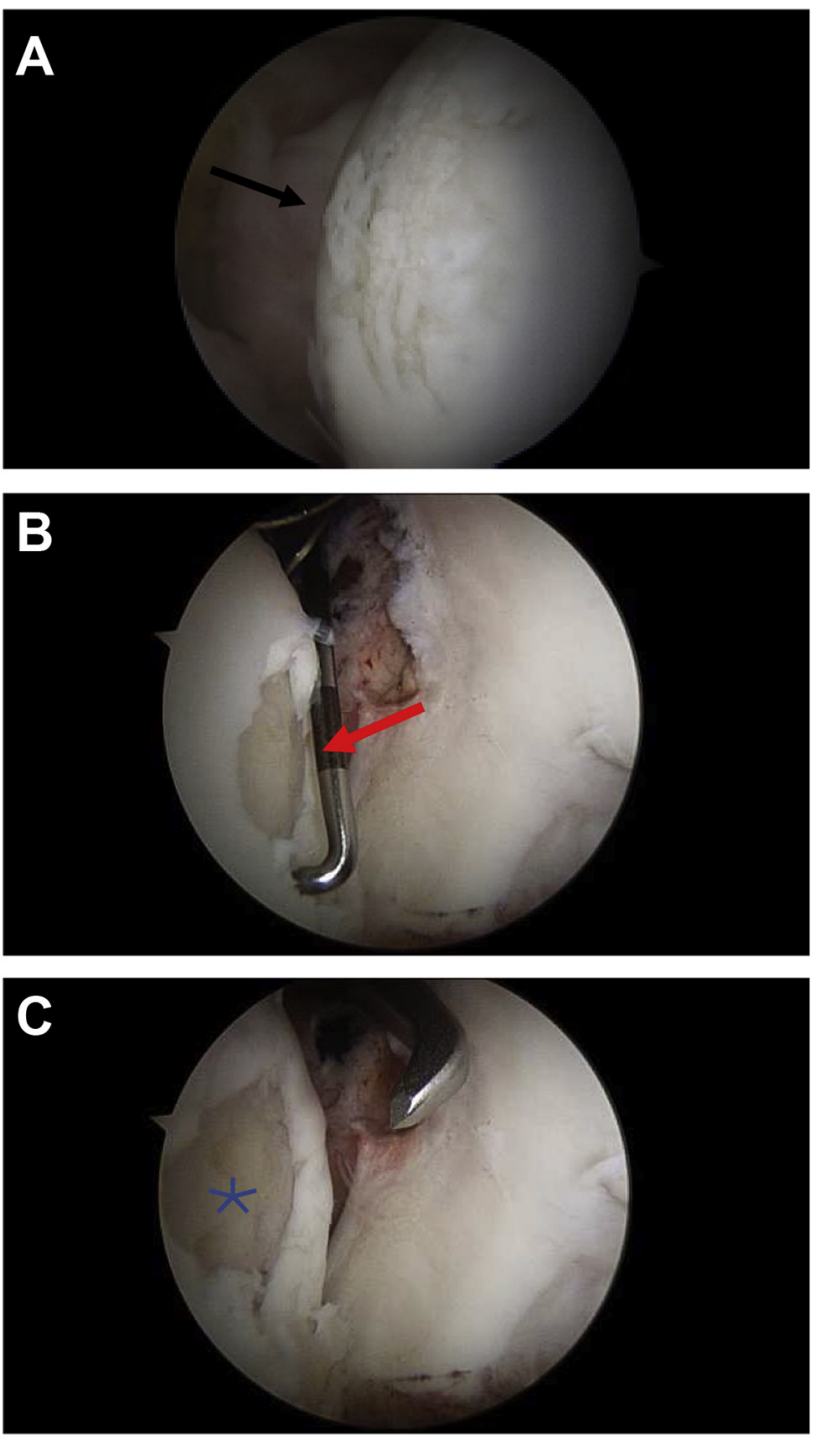

Fig 2. Intraoperative images of two patients demonstrating central head chondromalacia. (A) Right hip in the supine position viewing from a posterolateral view portal demonstrating an area of chondral softening (black arrow). (B) and (C) Images of a left hip viewed from a posterolateral portal with the acetabulum on the right and the femoral head on the right. The probe and microfracture awl are seen entering from the anterior portal. This demonstrates a central femoral head lesion after debridement (red arrow) and microfracture (star). 
Table 1. Demographic Data

\begin{tabular}{lccccc}
\hline & Non-Central & Central & \\
& CM $(n=32)$ & CM $(n=32)$ & $P$ Value \\
\hline Gender $(n, \%)$ & 9 & $28 \%$ & 24 & $75 \%$ & $<.001$ \\
$\quad$ Female & 23 & $72 \%$ & 8 & $25 \%$ & \\
$\quad$ Male & & & & & \\
Side $(n, \%)$ & 21 & $66 \%$ & 11 & $34 \%$ & .024 \\
$\quad$ Left & 11 & $34 \%$ & 21 & $66 \%$ & \\
$\quad$ Right & 39.2 & 10.6 & 40.7 & 13.5 & .623 \\
Age at surgery (mean, SD) & 39.7 & &
\end{tabular}

CM, chondromalacia; SD, standard deviation.

allowing full view of the femoral head (Fig 2). Imaging was reviewed to identify lateral center edge angle, $\alpha$ angle, and magnetic resonance imaging interpretation of femoral head chondromalacia, as available. The diagnosis of Cam-type FAI was defined by an $\alpha$-angle of greater than $55^{\circ}$. The diagnosis of pincer-type FAI was defined by a radiographic crossover sign and/or a lateral center edge of Wiberg of greater than $35^{\circ}$. Mixed FAI was defined by meeting criteria for both Cam and Pincer FAI. Ease of distractibility was determined intraoperatively on the basis of previously defined characteristics, including the amount of traction required for hip distraction and residual subluxation after release of traction. ${ }^{5}$

Chi-squared tests and Fisher's exact tests were used to analyze the rates of central femoral head CM by gender, race, side, and other diagnoses. A two-sample $t$-test was used to analyze the age at surgery for central and noncentral CM patients. All analyses were completed in RStudio version 1.1.456 (Boston, MA) using a twosided level of significance of .05.

\section{Results}

A total of 648 hip arthroscopies were performed during the study period. Seventy-four of these were for femoral head chondromalacia. We excluded 10 cases for the following reasons: 1 was a duplicate, 4 did not have femoral head chondromalacia, 1 had avascular necrosis, 1 had multiple epiphyseal dysplasias, 2 had femoral head cysts that required grafting, and 1 was diagnosed with pigmented villonodular synovitis. This left a total of 64 hips for review. Thirty-two of these had non-central head chondromalacia and 32 had central head chondromalacia (Table 1). Females were more likely than males to have central head chondromalacia, and this reached statistical significance $(P<.001)$.

Thirty-two patients were identified with central head chondromalacia, of whom, 26 had instability. Instability was isolated in 6 cases (two of which were revisions), 11 had instability with Cam-type FAI, 4 had instability with pincer-type FAI, 5 had instability with mixed-type FAI (1 revision), 1 CAM-type FAI, 4 mixed-type FAI, and 1 frozen hip. Frozen hip was a clinical diagnosis defined by significant decreased preoperative hip range of motion, particularly external rotation, and often also decreased flexion.

Central femoral head chondromalacia was associated with a diagnosis of instability, and this reached statistical significance (Table 2). Of the patients identified to have central head chondromalacia, $81 \%$ were found to have a diagnosis of hip instability. In contrast, of those who were identified to have non-central head chondromalacia, only $16 \%$ were identified to have instability. For patients with femoral head chondromalacia in the absence of instability, they were more likely to have mixed-type FAI ( $58 \%$ vs $23 \% ; P=.009$ ). Sensitivity, specificity, positive predictive values, and negative predictive values were calculated as outlined in Table 3.

\section{Discussion}

This study demonstrates that central head chondromalacia is associated with a diagnosis of hip microinstability. In patients identified as having central head chondromalacia at arthroscopy, $81 \%$ of those were found to have a diagnosis of hip instability, with $19 \%$ of those having isolated instability. Moreover, in this cohort of chondromalacia patients, the presence of central head chondromalacia was associated with an $84 \%$ sensitivity, $82 \%$ specificity, and $81 \%$ positive predictive value for the diagnosis of microinstability. These results suggest that chondromalacia at the

Table 2. Diagnoses Associated with Noncentral Versus Central Head Chondromalacia

\begin{tabular}{|c|c|c|c|c|c|}
\hline \multirow{2}{*}{$\overline{\text { Instability Dx }(n, \%)}$} & \multicolumn{2}{|c|}{$\begin{array}{l}\text { Non-Central } \\
\text { CM }(n=32)\end{array}$} & \multicolumn{2}{|c|}{$\begin{array}{c}\text { Central } \\
\text { CM }(n=32)\end{array}$} & \multirow[t]{2}{*}{$P$ Value } \\
\hline & & & & & \\
\hline No & 27 & $84 \%$ & 6 & $19 \%$ & $<.001$ \\
\hline Yes & 5 & $16 \%$ & 26 & $81 \%$ & \\
\hline \multicolumn{6}{|c|}{ Cam Dx $(n, \%)$} \\
\hline No & 20 & $63 \%$ & 20 & $63 \%$ & $>.999$ \\
\hline Yes & 12 & $38 \%$ & 12 & $38 \%$ & \\
\hline \multicolumn{6}{|c|}{ Pincer Dx $(n, \%)$} \\
\hline No & 30 & $94 \%$ & 28 & $88 \%$ & .672 \\
\hline Yes & 2 & $6 \%$ & 4 & $13 \%$ & \\
\hline \multicolumn{6}{|c|}{ Mixed Dx $(n, \%)$} \\
\hline No & 15 & $47 \%$ & 23 & $72 \%$ & .075 \\
\hline \multirow[t]{2}{*}{ Yes } & 17 & $53 \%$ & 9 & $28 \%$ & \\
\hline & \multicolumn{2}{|c|}{$\begin{array}{c}\text { No Instability } \\
\quad(n=33)\end{array}$} & \multicolumn{2}{|c|}{$\begin{array}{l}\text { Instability } \\
(n=31)\end{array}$} & $P$ Value \\
\hline \multicolumn{6}{|c|}{ Cam Dx $(n, \%)$} \\
\hline No & 21 & $64 \%$ & 19 & $61 \%$ & $>.999$ \\
\hline Yes & 12 & $36 \%$ & 12 & $39 \%$ & \\
\hline \multicolumn{6}{|c|}{ Pincer Dx $(n, \%)$} \\
\hline No & 32 & $97 \%$ & 26 & $84 \%$ & .099 \\
\hline Yes & 1 & $3 \%$ & 5 & $16 \%$ & \\
\hline \multicolumn{6}{|c|}{ Mixed Dx $(n, \%)$} \\
\hline No & 14 & $42 \%$ & 24 & $77 \%$ & .009 \\
\hline Yes & 19 & $58 \%$ & 7 & $23 \%$ & \\
\hline
\end{tabular}

Dx, diagnosis. 
Table 3. Sensitivity, Specificity, Positive, and Negative Predictive Values for the Diagnosis of Hip Microinstability

\begin{tabular}{lccccc}
\hline & Instability & No Instability & & & $95 \%$ Confidence Interval \\
\hline & & & Sensitivity & $84 \%$ & $(71 \%-97 \%)$ \\
Central CM & 26 & 6 & Specificity & $82 \%$ & $(69 \%-95 \%)$ \\
Noncentral CM & 5 & 27 & PPV & $81 \%$ & $(68 \%-95 \%)$ \\
& & & NPV & $84 \%$ & $(72 \%-97 \%)$ \\
\hline
\end{tabular}

center of the femoral head may contribute to the intraoperative diagnosis and identification of hip microinstability.

Microinstability is increasingly gaining support as an accepted cause of young, nonarthritic hip pain. As we improve our understanding of the altered kinematics, we are able to identify patterns associated with hip microinstability. To that end, others have identified injury and movement patterns associated with the diagnosis of hip microinstability. Clinically, Rosinsky et al. described a femoral head divot sign in 14 patients, identifying a linear chondral indentation on the femoral head just lateral and parallel to the acetabular labrum in patients identified to have hip microinstability. ${ }^{10}$ They proposed that, in the setting of microinstability, this area may experience repetitive edge loading leading to the head deformation.

Our study demonstrated that central femoral head chondromalacia was associated with a diagnosis of hip microinstability. One explanation for this phenomenon would be increased movement of the femoral head relative to the acetabulum in patients with microinstability, leading to central femoral head wear, possibly as it subluxates to, or over, the posterior acetabular rim or posterior cotyloid fossa. Movement of the central femoral head relative to the acetabulum has been identified and demonstrated to increase with increasing incompetence of the surrounding soft tissues in cadaveric models. ${ }^{12}$ Similarly, in vivo studies have demonstrated femoral head translations of .69-4.1 mm during hip range of motion. ${ }^{17,18}$ Considering these models and the intraoperative findings described in this study, it would seem intuitive that the central femoral head translation and, therefore, abnormal cartilage stresses and wear, would be amplified in the setting of microinstability.

Multiple different findings have been described to aid in the diagnosis of hip microinstability. However, to date, there is no consensus on diagnostic criteria for hip instability. Physical examination findings have been used to define patients with hip instability, with the abduction hyperextension external rotation test having the highest accuracy. ${ }^{3,9}$ Moreover, when a triad of tests-abduction hyperextension external rotation, abduction-extension external rotation, and prone external rotation-were found to be positive, it represented a $95 \%$ likelihood of hip instability identified at the time of surgery. ${ }^{9}$ Generalized ligamentous laxity has been found to be associated with higher rates of labral repair and capsular plication, but it has not been found to correlate strongly with microinstability. $3,8,11$ Although many radiographic signs have been evaluated, a recent systemic review failed to find strong evidence for any diagnostic radiographic test and concluded that radiographic diagnosis must be in the context of clinical diagnosis. ${ }^{4}$ Finally, intraoperative criteria, which have been suggested to include ease of distractibility, location of acetabular chondral damage, and location of the acetabular labral tear. ${ }^{5,19}$ Shibata et al. found that there was a predilection for straight anterior and lateral acetabular chondral or labral damage in patients with hip microinstability. ${ }^{5}$

Considering the evidence, our current understanding of hip microinstability requires a compilation of history, clinical exam, radiographic studies, and intraoperative findings to make the diagnosis. As such, the primary purpose of this study was to help add an additional diagnostic tool to aid in the clinical diagnosis of hip microinstability. This article demonstrated that central femoral head chondromalacia was identified in a high percentage of patients found to have hip microinstability. When added to the other diagnostic findings above, this may aid the surgeon in intraoperative decision making, particularly, when it comes to capsular management and consideration of capsular plication. As we continue to expand our understanding of the multifaceted diagnostic tools, we will further expand our understanding of elements of the history, physical exam, imaging studies, and intraoperative findings, allowing us to further delineate objective findings associated with hip microinstability to inform patientspecific treatment.

\section{Limitations}

This study is not without limitations. First, it is retrospective in nature and is inherently limited by its retrospective design. Second, there is currently no universally accepted diagnostic criteria for hip microinstability, so we relied on previously defined and published criteria in this study. The diagnosis of hip microinstability in this cohort was based on a combination of preoperative history and physical, radiographic findings, and intraoperative findings associated with hip microinstability, as determined by the senior surgeon. This leaves open the possibility of observer bias in identifying these patients as having microinstability. Third, there was a significant association found between instability and gender, raising 
concern for confirmation bias for the diagnosis of instability. Although we are unable to account for this type of bias, it should be noted that much of the literature on microinstability report a high percentage of instability patients are female. Fourth, we found a statistically significant difference in central versus noncentral wear based on laterality. We are unable to account for this difference as the same technique is used for hip arthroscopy, irrespective of laterality. Finally, we included revision cases in our analysis, and this may conflate our analysis. We feel that this is mitigated by the small percentage of our total cases being revisions $(<5 \%)$ and the mixed location of the chondral damage ( 2 central, 1 noncentral).

\section{Conclusion}

A high percentage of patients with central femoral head chondromalacia were found to have hip microinstability. These results suggest that there is a pattern of femoral head chondral damage in patients with hip microinstability.

\section{References}

1. Dangin A, Tardy N, Wettstein M, May O, Bonin N. Microinstability of the hip: A review. Orthop Traumatol Surg Res 2016;102:S301-S309.

2. Parvaresh KC, Rasio J, Azua E, Nho SJ. Hip instability in the athlete: Anatomy, etiology, and management. Clin Sports Med 2021;40:289-300.

3. Safran MR. Microinstability of the hip-gaining acceptance. J Am Acad Orthop Surg 2019;27:12-22.

4. Woodward RM, Vesey RM, Bacon CJ, White SG, Brick MJ, Blankenbaker DG. Microinstability of the hip: A systematic review of the imaging findings. Skeletal Radiol 2020;49:1903-1919.

5. Shibata KR, Matsuda S, Safran MR. Is there a distinct pattern to the acetabular labrum and articular cartilage damage in the non-dysplastic hip with instability? Knee Surg Sports Traumatol Arthrosc 2017;25:84-93.

6. Kalisvaart MM, Safran MR. Hip instability treated with arthroscopic capsular plication. Knee Surg Sports Traumatol Arthrosc 2017;25:24-30.

7. Domb BG, Stake CE, Lindner D, El-Bitar Y, Jackson TJ. Arthroscopic capsular plication and labral preservation in borderline hip dysplasia: Two-year clinical outcomes of a surgical approach to a challenging problem. Am J Sports Med 2013:41:2591-2598.

8. Saadat AA, Lall AC, Battaglia MR, Mohr MR, Maldonado DR, Domb BG. Prevalence of generalized ligamentous laxity in patients undergoing hip arthroscopy: A prospective study of patients' clinical presentation, physical examination, intraoperative findings, and surgical procedures. Am J Sports Med 2019;47:885-893.

9. Hoppe DJ, Truntzer JN, Shapiro LM, Abrams GD, Safran MR. Diagnostic accuracy of 3 physical examination tests in the assessment of hip microinstability. Orthop $J$ Sports Med 2017;5:2325967117740121.

10. Rosinsky PJ, Mayo BC, Kyin C, et al. The femoral head "divot" sign: A useful arthroscopic sign of hip microinstability. Orthop J Sports Med 2020;8:2325967120917919.

11. Han S, Alexander JW, Thomas VS, et al. Does capsular laxity lead to microinstability of the native hip? Am J Sports Med 2018;46:1315-1323.

12. Safran MR, Lopomo N, Zaffagnini S, et al. In vitro analysis of peri-articular soft tissues passive constraining effect on hip kinematics and joint stability. Knee Surg Sports Traumatol Arthrosc 2013;21:1655-1663.

13. Signorelli C, Lopomo N, Bonanzinga T, et al. Relationship between femoroacetabular contact areas and hip position in the normal joint: an in vitro evaluation. Knee Surg Sports Traumatol Arthrosc 2013;21:408-414.

14. Mitchell RJ, Gerrie BJ, McCulloch PC, et al. Radiographic evidence of hip microinstability in elite ballet. Arthroscopy 2016;32:1038-1044 e1031.

15. Charbonnier C, Kolo FC, Duthon VB, et al. Assessment of congruence and impingement of the hip joint in professional ballet dancers: a motion capture study. Am J Sports Med 2011 ; 39:557-566.

16. Duthon VB, Charbonnier C, Kolo FC, et al. Correlation of clinical and magnetic resonance imaging findings in hips of elite female ballet dancers. Arthroscopy 2013;29:41 1-419.

17. Kolo FC, Charbonnier C, Pfirrmann CW, et al. Extreme hip motion in professional ballet dancers: Dynamic and morphological evaluation based on magnetic resonance imaging. Skeletal Radiol 2013;42:689-698.

18. Kapron AL, Aoki SK, Peters CL, Anderson AE. In-vivo hip arthrokinematics during supine clinical exams: Application to the study of femoroacetabular impingement. J Biomech 2015:48:2879-2886.

19. Boykin RE, Anz AW, Bushnell BD, Kocher MS, Stubbs AJ, Philippon MJ. Hip instability. J Am Acad Orthop Surg $2011 ; 19: 340-349$. 